\title{
El acompañamiento de las trayectorias académicas estudiantiles: experiencia de seguimiento desde el lugar de la adscripción en la cátedra de Didáctica de la FaHCE - UNLP
}

\section{The accompaniment of student academic trajectories: monitoring experience from the place of assignment in the Didactics chair FaHCE - UNLP}

\section{Elisa Marchese}

https://orcid.org/0000-0001-5866-7324

elilaca@hotmail.com

Facultad de Humanidades y Ciencias de la Educación | Universidad Nacional

de La Plata | Argentina

\author{
Juan Sebastián Allussón \\ https://orcid.org/0000-0001-5385-2475 \\ allusson.juan@gmail.com \\ Facultad de Humanidades y Ciencias \\ de la Educación | Universidad Nacional \\ de La Plata | Argentina
}

\section{RESUMEN}

En el presente trabajo, pretendemos a la vez relatar y analizar la experiencia de acompañamiento de las trayectorias académicas de estudiantes que cursaron oportunamente la materia Didáctica y que aún, por diversos motivos, adeudan la acreditación del examen final.

La iniciativa consiste en diseñar e implementar una serie de estrategias tendientes a acompañar al grupo de estudiantes que se encuentra en esta situación para que puedan finalmente acreditar la asignatura y proseguir su recorrido académico.

Como estrategia, confeccionamos la nómina de las personas que cursaron la materia entre los años 2015-2020, para identificar si aprobaron la cursada y corroborar si ya promovieron el final de la materia. En dicha nómina, además contamos con información de contacto de estudiantes para entablar una comunicación y a partir de allí, generar lo que llamamos "Mateadas Prefinales" que son espacios de acompañamiento y orientación en donde los y las estudiantes puedan plantear las necesidades, inquietudes y dificultades que se les presentan a la hora de prepararse para rendir el examen final de Didáctica.

Esperamos que este artículo posibilite profundizar nuestra reflexión permanente como cátedra sobre las prácticas de enseñanza así como contribuir al mejoramiento de estrategias de acompañamiento de las trayectorias estudiantiles y de accesibilidad al derecho a la educación superior.

\section{PALABRAS CLAVE}

Enseñanza, Estrategias de Acompañamiento, Evaluación, Adscripción. 
KEY WORDS

Teaching,

Accompaniment Strategies,

Evaluation,

Secondments Membership.

\section{ABSTRACT}

In the present work, we intend to report and analyze the experience of monitoring the academic trajectories of students who took the Didactics subject in a timely manner and who still, for various reasons, owe the accreditation of the final exam.

The initiative consists of designing and implementing a series of strategies aimed at accompanying the group of students who find themselves in this situation so that they can finally accredit the subject and continue their academic way.

As a strategy, we first prepare the payroll of the people who took the course between 2015 and 2020, to identify if they passed the course and corroborate if they have already promoted the end of the course. In this list, we also have contact information for students to initiate communication and from there, generate what we call "Mateadas Prefinales" which are support and orientation spaces where students can raise their needs, concerns and difficulties that arise when preparing to take the final exam in Didactics

We hope that this article will make it possible to deepen our permanent reflection as a chair on teaching practices as well as to contribute to the improvement of strategies for accompanying student trajectories and accessibility to the right to higher education. 


\section{PRESENTACIÓN DE LA EXPERIENCIA}

La materia Didáctica se encuentra en el segundo año del Plan de Estudio de las carreras del Profesorado y Licenciatura en Ciencias de la Educación de la Facultad de Humanidades y Ciencias de la Educación (FaHCE-UNLP). Como materia obligatoria es parte de la formación básica de ambas carreras. Desde la propuesta formativa de la cátedra procuramos ahondar en los aspectos teórico-conceptuales propios de esta disciplina que forma parte del campo pedagógico. Entendemos la "Didáctica como un campo disciplinar diverso conformado por teorías que se ocupan de estudiar la enseñanza" (programa 2020) y que pretende la comprensión de esta práctica social compleja así como la intervención de manera más ajustada a la realidad de los ámbitos en donde se desarrollan las prácticas formativas. En este marco, entendemos que lo contextual se erige como un factor clave para la comprensión de los escenarios de actuación docente.

La metodología de trabajo es un aspecto relevante y explicativo de esta experiencia. En ese sentido, desde la cátedra proponemos y desarrollamos actividades que propician la puesta en práctica de una evaluación continua, permanente y formativa que incluye a quienes, de una manera u otra, participan de la cursada de la materia (docentes, estudiantes, adscritos/as). A este respecto los intercambios y debates que promovemos en los distintos escenarios de intervención (clases teóricas, clases prácticas, reuniones de la cátedra, mateadas pre coloquio, mateadas pre finales) se convierten en el necesario punto de encuentro desde donde construimos nuevos saberes que redundan en más y mejores prácticas de intervención. En consonancia con esta dinámica es que hemos asumido que el acompañamiento de las trayectorias estudiantiles es uno de los aspectos primordiales del trabajo 
de cátedra. Testimonios de esta labor son los diferentes espacios que históricamente han estado habilitados para tal fin: clases de consulta y tutorías presenciales, así como el correo electrónico de la cátedra que ponemos a disposición para que quienes cursan o adeudan el final, puedan comunicarse.

La experiencia que compartimos en esta oportunidad y que protagonizamos quienes escribimos este artículo como parte de nuestro proyecto de adscripción a la cátedra, constituye una nueva propuesta superadora de las instancias anteriores en tanto acompañamiento más cercano de las trayectorias académicas estudiantiles. Es así que a principios de 2020 elaboramos este proyecto de adscripción a los fines de lograr un seguimiento más ajustado a las inquietudes y dificultades que presentan los/as estudiantes que han cursado la materia en el período $2015^{2}-2020$ y que desean rendir el examen final pero que por diversos motivos no lo han hecho aún. Lo antedicho es indicativo de que nuestra mirada está atenta a las particularidades de las necesidades de los/as estudiantes y que son propias de cada trayectoria individual.

Siguiendo a Terigi (2010), entendemos que este acompañamiento es posible a partir del reconocimiento de las trayectorias educativas reales. Las trayectorias teóricas representan el recorrido académico esperado según la progresión lineal prevista por el Sistema Educativo o el Plan de Estudio de la carrera en este caso. Por su parte, las trayectorias educativas reales son expresiones de condiciones socioeducativas diversas que atraviesan la vida académica de quienes cursan hoy en la Universidad, jalonadas por tiempos diferentes, por situaciones laborales y familiares diversas y que desatendidas, pueden conducir a la discontinuidad, el ausentismo o al abandono de los estudios.

Entendemos que las estrategias singulares que podamos diseñar e implementar como cátedra para el acompañamiento real de las trayectorias estudiantiles, son las que integrarán y fortalecerán un conjunto más amplio de decisiones de política institucional tendientes a la real concreción del acceso, permanencia y egreso de cada estudiante en la educación superior universitaria.

\section{DESCRIPCIÓN DE LA EXPERIENCIA}

El relato de la experiencia, iniciada a principios de 2020, es el de una actividad que llevamos adelante entre el equipo docente de la cátedra con la participación de dos personas adscriptas a la misma. Según la Resolución 653/08 sobre el Sistema de Adscripciones a la Docencia Universitaria en el ámbito de la UNLP "se promueve el acercamiento integral del alumno/a y/o graduado/a a la vida académica universitaria a partir de su inclusión en una cátedra específica y de su parti- 
cipación en las actividades y roles que esta actividad demanda, a fin de fortalecer su formación profesional" (Res. 653/08, art.1) ${ }^{3}$. En otros términos, las adscripciones fortalecen la idea de que la cátedra universitaria es un espacio de formación en múltiples sentidos, en este caso de colegas docentes que apuestan a una formación permanente y que intervienen en diferentes niveles del sistema educativo. No es menor mencionar que las adscripciones son espacios de desempeño no rentados al interior de las cátedras donde, en algunos casos como el de nuestra experiencia, el desarrollo de las actividades son de docencia.

Desde que la cátedra tiene la actual constitución de la planta docente (año 2015), creamos una base de datos en donde fuimos recopilando y organizando la información de los/as estudiantes que cursaron la materia. Cabe mencionar que en promedio se inscriben a la cursada entre 50 y 70 estudiantes por año. En esta base de datos fuimos consignando: año de cursada, dirección de correo electrónico ${ }^{4}$, calificación obtenida al aprobar la cursada y si la materia se encuentra o no acreditada completamente.

Asimismo, el Régimen de Enseñanza y Promoción (REP en adelante) ${ }^{5}$ de la FaHCE-UNLP indica en su artículo 8: "Las materias de la Facultad ofrecerán los siguientes sistemas de promoción: a) Promoción con examen final libre; b) Promoción con cursada regular y examen final; c) Promoción con cursada regular, descarga parcial de contenidos y examen final reducido; d) Promoción sin examen final". En el caso de Didáctica prevemos la evaluación y acreditación de la materia considerando las cuatro modalidades propuestas en el REP. Para ello, en el programa de la asignatura, hemos especificado las condiciones de promoción en cada caso según corresponda y construimos materiales específicos para acompañar cada una de las instancias de evaluación.

Para la preparación del examen final regular, los/as estudiantes deben desarrollar una producción integradora de los contenidos de las cinco unidades que conforman el programa de Didáctica. Para ello, fundamentalmente deben realizar la lectura con el correspondiente análisis reflexivo de la bibliografía obligatoria. Existen diferentes alternativas para la realización de la producción integradora: cuadros, esquemas o mapas conceptuales, construcción de alguna maqueta, realización de dibujos, producciones digitales u otra opción que los y las estudiantes consideren adecuada para lograr articular, vincular e integrar los contenidos explicitados en el programa de la materia.

\footnotetext{
3 Reglamento de Adscripciones de la FaHCE-UNLP. Resolución 653/08 http://www.fahce.unlp. edu.ar/normativa/reglamento-de-adscripciones-resolucion-653-2008

4 Dato que en algunas oportunidades tuvimos que solicitar a compañeros/as o al Departamento de Ciencias de la Educación FaHCE-UNLP para completar la información por no contar con ella. 
Para la preparación del examen final regular, los/as estudiantes deben desarrollar una producción integradora de los contenidos de las cinco unidades que conforman el programa de Didáctica. Para ello, fundamentalmente deben realizar la lectura con el correspondiente análisis reflexivo de la bibliografía obligatoria. Existen diferentes alternativas para la realización de la producción integradora: cuadros, esquemas o mapas conceptuales, construcción de alguna maqueta, realización de dibujos, producciones digitales u otra opción que los y las estudiantes consideren adecuada para lograr articular, vincular e integrar los contenidos explicitados en el programa de la materia.

El examen final integrador es de carácter oral, individual. Consiste en explicar las pautas y criterios que fundamentan las decisiones que operaron en la realización de la producción integradora, así como el desarrollo conceptual de algún núcleo de esa producción y dar respuesta a las preguntas formuladas por las docentes que conforman la cátedra acerca de los contenidos de la materia (especificados en el programa correspondiente para el examen).

Cuando los/as estudiantes han avanzado en la lectura de la bibliografía y han podido proyectar una idea de la producción integradora acordamos horarios de consulta presenciales o virtuales (en la actualidad sólo se ofrece la segunda opción por las medidas de aislamiento/distanciamiento decretadas por el gobierno nacional a causa de la pandemia por el COVID-19) a los fines de ofrecerles orientación para que puedan avanzar en sus producciones, atender inquietudes y despejar dudas.

Cuando los/as estudiantes han avanzado en la lectura de la bibliografía y han podido proyectar una idea de la producción integradora acordamos horarios de consulta presenciales 0 virtuales (en la actualidad sólo se ofrece la segunda opción por las medidas de aislamiento/distanciamiento decretadas por el gobierno nacional a causa de la pandemia por el COVID-19) a los fines de ofrecerles orientación para que puedan avanzar en sus producciones, atender inquietudes y despejar dudas..

Además hemos confeccionado una guía de contenidos denominada "Temas centrales". Hay una guía disponible por cada uno de los programas que se han ido implementando a lo largo de estos años, dirigida a los/as estudiantes con el propósito de que actúe como un instrumen- 
to de autoevaluación de los avances de sus producciones integradoras y de los propios aprendizajes. La guía en cuestión consta de una serie de cuadros a dos columnas, uno por cada unidad. En la columna ubicada a la izquierda figuran los contenidos y objetivos mínimos y en la de la derecha, se encuentra la bibliografía o los materiales desde los cuales abordar dichos contenidos así como una posible secuencia para realizar las lecturas (ver ejemplo en el Anexo). Es una guía de autoevaluación que posibilita a quienes tienen que rendir el final poder ir evaluando su proceso de estudio así como sus conocimientos antes de presentarse a la instancia evaluadora. Constituye una herramienta valiosa en tanto explicita y democratiza los criterios de evaluación que desde la cátedra utilizamos para valorar los aprendizajes de quienes pretenden acreditar el final de la materia. Complementariamente, estudiantes que participan de la cátedra como adscriptas o colaboradoras, realizaron una serie de videos explicativos sobre cómo ellas resolvieron la construcción de esta actividad integradora para acreditar la materia ${ }^{6}$.

En la experiencia ocupan un lugar central los encuentros de acompañamiento, que denominamos "Mateadas Pre Finales", abiertas tanto a personas que han aprobado la cursada y deben rendir el examen final (modalidad b) como también a quienes lo deben hacer en forma libre (modalidad a, artículo 8, del REP).

En el marco de este trabajo de acompañamiento, pretendemos específicamente recuperar la formación como trayectoria, para lo cual "es necesario interrogarse por los hilos que permiten hilvanar las numerosas y diversas experiencias por las que el estudiante transita" (Nicastro y Greco, 2009, p.54). Se trata de tender puentes, de crear un espacio de acompañamiento que interviene mediando entre las condiciones reales y la posibilidad de un hacer "a medida" que es pensando colectivamente. Las estrategias desplegadas a partir de la información de la base de datos se convirtieron en nuestros "puentes" para realizar los contactos con los/as estudiantes.

En el marco de este trabajo de acompañamiento, pretendemos específicamente recuperar la formación como trayectoria, para lo cual "es necesario interrogarse por los hilos que permiten hilvanar las numerosas y diversas experiencias por las que el estudiante transita" (Nicastro y Greco, 2009, p.54). Se trata de tender puentes, de crear un espacio de acompañamiento que interviene mediando entre las condiciones reales y la posibilidad de un hacer "a medida" que es pensando colectivamente.

\footnotetext{
6 Disponibles en: https://drive.google.com/file/d/1sj8fQ0dkOd2YcIXMYHosSnne66DdmvLc/ view https://drive.google.com/file/d/1AkLc-tyAUyupTP09i4JNOV1FWeLUmYJe/view https://drive.google.com/file/d/1IUL7oyZSGmdKJUkrtV8sYjgyGDsyj3RN/view
} 
Adentrándonos en el detalle de las estrategias de acompañamiento que venimos desarrollando, podemos decir que la secuencia de pasos que seguimos son las siguientes:

$1^{\circ}$ Momento: siguiendo el calendario académico de la FaHCE-UNLP, durante el período de inscripción a las mesas de finales nos comunicamos con quienes adeudan el examen final vía correo electrónico. Les informamos de la vigencia del período de inscripciones e invitamos a quienes tengan intenciones de rendir que lo hagan, anticipando que realizaremos algunas actividades que pretenden acompañar la construcción del examen.

$2^{\circ}$ Momento: una vez terminado el período de inscripción accedemos a la nómina de estudiantes que se inscribieron para rendir. Luego consultamos en nuestros registros para constatar si es estudiante que rinde en condición libre o regular, en qué año cursó la materia y si tiene acceso al campus ${ }^{7}$ del año correspondiente y a los materiales allí disponibles.

En caso que corresponda, acompañamos el proceso de Solicitud de Reválida ${ }^{8}$ de la asignatura contemplada en el REP. Dicho trámite implica la presentación de una nota en el Departamento de nuestra carrera, donde el/la estudiante indique el año de cursada, sus datos personales y de contacto y formulen la petición a la cátedra.

$3^{\circ}$ Momento: realizamos una segunda comunicación con quienes se inscribieron para presentar con un poco más de detalle el espacio de la Mateada Prefinales socializando nuestro propósito, anticipando las actividades a realizar como así también, las fechas importantes a considerar. Allí pedimos que puedan asistir con alguna idea, esquemas, bosquejos de la producción a presentar en el final para poder trabajar de modo concreto sobre ello. Enviamos la invitación a asistir junto con el día y horario propuesto como así también el link de acceso a la videollamada de la Mateada Pre Finales.

En caso que sea necesario, enviamos un correo con dos archivos adjuntos: el primero con el programa correspondiente para rendir el examen y el documento que contiene los temas centrales del programa; el segundo archivo explica la modalidad del examen final junto con los criterios de evaluación.

$4^{\circ}$ Momento: destinamos los primeros instantes de la Mateada a corroborar año y modalidad de evaluación que corresponda. Además atendemos consultas y resolvemos los problemas vinculados al acceso a materiales, al campus, relevamos si hay consultas sobre la modalidad de la evaluación. Luego invitamos a los/as estudiantes a compar-

$7 \quad$ Plataforma de la Facultad de Humanidades y Ciencias de la Educación en donde se encuentran las Aulas Virtuales de cursadas de materias, seminarios y talleres de grado de todas las carreras de la Facultad, organizadas por Departamento Docente. 
tir sus avances en torno a la construcción de la producción; a partir de esta presentación, vamos formulando preguntas e intercambios entre pares. Allí también acordamos avances en torno a sus producciones que atiendan a las sugerencias formuladas y la fecha del próximo encuentro.

Posteriormente, y hasta el momento del examen final, realizamos un seguimiento permanente, mediante la comunicación por correo electrónico y en caso que sea necesario, de otros encuentros sincrónicos, donde compartimos avances en las lecturas y la apropiación de contenidos de quienes se encuentran en la instancia de preparar el examen. También enviamos diversos materiales ya producidos en años anteriores así como otros nuevos que van surgiendo en este contexto obligado de virtualización de la enseñanza, entre ellos: guías de estudio, diapositivas de clase, actividades, videos explicativos..

Como se puede observar, desde la cátedra desarrollamos este "hacer a medida" pensando a los/as estudiantes con sus particularidades, realidades sociales y laborales. En este sentido, el trabajo de seguimiento, orientación y acompañamiento a quienes cursaron la asignatura y adeudan aún la acreditación del examen final ha obtenido muy buenas respuestas ya que estas nuevas condiciones de confinamiento para ralentizar la circulación del COVID-19 significan para algunos/as tener otros tiempos disponibles para dedicar al estudio.

\section{CONCLUSIONES}

La escena educativa en el presente contexto de pandemia se ha tornado más compleja puesto que se han profundizado transformaciones que previamente estaban en ciernes y además han incrementado las desigualdades socioculturales y económicas. Estas nuevas condiciones tienen a priori una incidencia negativa en las trayectorias educativas de los/as estudiantes. En ese sentido y en consonancia con las políticas de inclusión promovidas desde la Universidad hemos asumido un posicionamiento ante esta problemática que sitúa al derecho a la educación superior en un primer plano.

Algunas ideas asociadas con el posicionamiento asumido, frente a este contexto complejo y problemático, han resultado relevantes a la hora de hacer una primera evaluación de nuestra experiencia. Al respecto, dos conceptos aparecen como cruciales y transversales a la hora de realizar un primer análisis: evaluación y trayectorias. Es que tanto las formas que asume la evaluación como la consideración acerca de lo que las trayectorias de los/as estudiantes significan hacia el interior de los procesos de enseñanza y de aprendizaje, dan cuenta de los posicionamientos éticos, políticos, pedagógicos y didácticos que sustentan nuestras intervenciones. En ese sentido, y coincidiendo con Santos Guerra (1996), reconocemos que la evaluación posee un fuerte 
componente sociológico que cumple una función de selección que, a manera de tamiz o filtro, favorece u obstaculiza el progreso de los/ as estudiantes en las posteriores etapas dentro del sistema educativo. Partiendo de esta premisa y del desarrollo de la experiencia es que hemos podido dimensionar la trascendencia y el impacto que poseen las prácticas evaluativas en las trayectorias educativas.

Siguiendo a Nicastro y Greco (2009), en cuanto a que las trayectorias educativas constituyen caminos a recorrer y a construir que agrupan a sujetos en situación de acompañamiento, es que podemos afirmar que en nuestro caso el escenario de las intervenciones docentes se amplía y se sitúa en otros nuevos territorios. Es así que los procesos de enseñanza y de aprendizaje se plantean como una co-responsabilidad de quienes participamos de la cursada. Ya no es el examen final una responsabilidad exclusiva de los/as estudiantes, es así que proponemos y promovemos acciones que consideren cada trayectoria con sus singularidades, acompañando a cada estudiante que aún no ha rendido el examen final. Subyace en la experiencia una perspectiva atenta a comprender las trayectorias del estudiantado de manera situada para así poder desarrollar estrategias de acompañamiento ajustadas que las dinamicen en aras de garantizar desde nuestra labor el derecho a la educación superior y bregar por una educación más inclusiva. Este dispositivo de permanencia y egreso, centrado en lo pedagógico-didáctico-curricular, es de importancia en términos de democratización de la universidad y, si bien no es el único aspecto del cual depende la trayectoria real de nuevos sectores de la población bajo condiciones de vulnerabilidad social, reafirma la demanda de otras acciones por parte de la enseñanza para garantizar el derecho a la educación superior. En este sentido, es que acordamos con Terigi (2004) en su propuesta de recuperar la idea de la enseñanza no sólo como problema didáctico sino también político, que exige el diseño y la implementación de políticas educativas que acompañen y sostengan este tipo de decisiones didácticas. 


\section{BIBLIOGRAFÍA}

Lenz, S. (2016). "Las universidades nacionales frente al desafío de la democratización: estrategias institucionales y dispositivos de acceso y permanencia". En Del Valle, D.; Montero, F.; y Mauro, S. (Comps.) El derecho a la Universidad en perspectiva regional. Buenos Aires: IEC CONADU, CLACSO.

Nicastro, S. y Greco, B. (2009). "Entre trayectorias. Escenas y pensamientos en espacios de formación". Rosario, Homo Sapiens.

Régimen de Enseñanza y Promoción, Facultad de Humanidades y Ciencias de la Educación, Universidad Nacional de La Plata, 2011 con modificaciones 2015. Recuperado de http://www.fahce.unlp.edu.ar/academica/regimen-de-ensenanza-y-promocion

Resolución n 653/08 Sobre el Sistema de Adscripciones de la FaHCE- UNLP.

Santos Guerra, M. Á. (1996). “Evaluar es comprender. De la concepción técnica a la dimensión crítica”. Revista Investigación en la Escuela, (30), 5-13. Recuperado de http://www.investigacionenlaescuela.es/index.php/revista-investigacion-en-laescuela/183-nmero_30_evaluar_no_es_calificar

Terigi, F. (2004). "La enseñanza como problema político". En Frigerio, G. y Diker, G. (Comps.) La transmisión en las sociedades, las instituciones y los sujetos. Un concepto de la Educación en acción. Ediciones Novedades Educativas. Buenos Aires. Recuperado de https://www.fapyd.unr.edu.ar/wp-content/uploads/2017/08/Terigi-la\%20ense\%C3\%B1 anza\%20como\%20problema\%20politico.pdf

Terigi, F. (2010). “Las cronologías de aprendizaje: un concepto para pensar las trayectorias escolares". Conferencia de apertura del ciclo lectivo 2010, Santa Rosa, La Pampa. Ministerio de Cultura y Educación de La Pampa. Recuperado de http://nuestraescuela.educacion.gov.ar/bancoderecursos/ media/docs/bloque03/Terigi_Flavia_Conferencia_Las_cronologias_de_ aprendizaje.pdf

\section{Anexo:}

Didáctica temas centrales programa 2020 Consiste en una síntesis que ofrecemos a quienes cursaron la materia con el programa 2020 y deben rendir el final. Específicamente consta de un listado de los contenidos que se deben saber para alcanzar los objetivos de la asignatura y la bibliografía que los aborda. Para cada unidad del programa se ofrece un cuadro: en la columna de la izquierda aparecen los contenidos y en la columna de la derecha la bibliografía desde la que abordarlos en un posible orden de lectura. Incluimos a modo de ejemplo el correspondiente a la unidad 1: 
Unidad 1: Enseñanza como objeto de estudio y de intervención.

\begin{tabular}{|c|c|}
\hline $\begin{array}{c}\text { Contenidos que deben saberse } \\
\text { para el alcance de los objetivos } \\
\text { del programa }\end{array}$ & Bibliografía desde la que estudiar los contenidos \\
\hline $\begin{array}{l}\text { Definición genérica de enseñanza: } \\
\text { aspectos constitutivos, } \\
\text { intencionalidad, relación ontológica. }\end{array}$ & 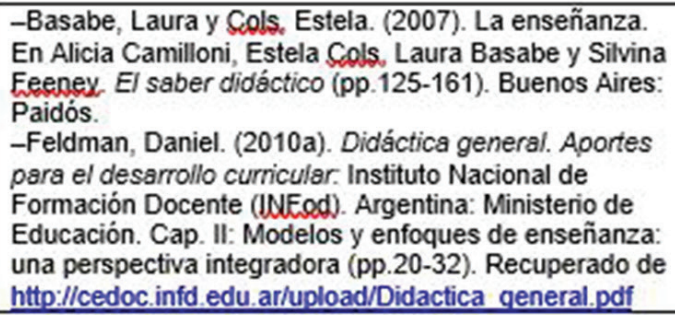 \\
\hline $\begin{array}{l}\text { Definición elaborada de enseñanza: } \\
\text { características. Buena enseñanza, } \\
\text { posicionamiento para definirla. } \\
\text { ¿En torno a qué dimensiones se } \\
\text { diferencian las definiciones } \\
\text { elaboradas de enseñanza? } \\
\text { Enfoques de enseñanza: } \\
\text { características principales de los tres } \\
\text { enfoques: ejecutivo, terapeuta y } \\
\text { liberador. Relación con la definición } \\
\text { elaborada de enseñanza. }\end{array}$ & $\begin{array}{l}\text {-Basabe, Laura y Cols, Estela. (2007). } \\
\text {-Feldman, Daniel. (2010a). }\end{array}$ \\
\hline $\begin{array}{l}\text { Concepciones del aprendizaje. } \\
\text { Complejidades de los procesos de } \\
\text { aprendizaje. Relación con las } \\
\text { estrategias de enseñanza. }\end{array}$ & $\begin{array}{l}\text {-Alliaud, Andrea. (2017). Los artesanos de la } \\
\text { enseñanza. Acerca de la formación de maestros con } \\
\text { oficio. Buenos Aires: Paidós. Cap. 1: Sobre las } \\
\text { instituciones: las escuelas ya no son lo que eran (pp.19- } \\
\text { 32) y cap. 2: Sobre la enseñanza: recuperar la } \\
\text { perspectiva del oficio (pp.33-55). } \\
\text {-Feldman. Daniel. (2010a). }\end{array}$ \\
\hline $\begin{array}{l}\text { Aspectos que integran la enseñanza. } \\
\text { La enseñanza en el ámbito escolar. } \\
\text { Las responsabilidades docentes con } \\
\text { respecto a la enseñanza. } \\
\text { Enseñanza y profesorado: } \\
\text { responsabilidades, márgenes de } \\
\text { actuación. }\end{array}$ & $\begin{array}{l}\text {-Alliaud, Andrea. (2017) } \\
\text {-Basabe, Laura y Sols, Estela. (2007). }\end{array}$ \\
\hline $\begin{array}{l}\text { Relaciones entre la teoría y la } \\
\text { práctica en la enseñanza y en la } \\
\text { Didáctica. La enseñanza como } \\
\text { praxis y poiesis. } \\
\text { La enseñanza como objeto de } \\
\text { estudio de la Didáctica, la Didáctica } \\
\text { como disciplina (introducción que se } \\
\text { profundiza en la unidad 5). }\end{array}$ & $\begin{array}{l}\text {-Rouzzo. Vilma. (2014). Didáctica genergl. Investigación } \\
\text { empírica y discusiones teóricas. Argentina: AMAX. Cap. } \\
\text { 2: La enseñanza, objeto de estudio de la Didáctica } \\
\text { (selección pp.37-44) y cap. 3: La enseñanza entre la } \\
\text { praxis y la poiesis, Aportes a la Didáctica general } \\
\text { (pp.55-78). Recuperado de } \\
\text { httos://unw. academia.edu/24020409/Did\%C3\%A1ctica } \\
\text { General. Investiqaci\%C3\%B3n empC3\%ADrica y } \\
\text { discusiones te\%C3\%B3ricas }\end{array}$ \\
\hline $\begin{array}{l}\text { Perspectiva multirceferencial para } \\
\text { estudiar la enseñanza. } \\
\text { El lugar de lo afectivo, lo emocional. }\end{array}$ & $\begin{array}{l}\text {-Mazza, Diana. (2015). La perspectiva clínica en } \\
\text { educación. Entrevista a Diana Mazza. Boletín de } \\
\text { Novedades Educativas } N^{\circ} 60 \text {. Recuperado de } \\
\text { https://unw.fundacionluminis.orq.ar/biblioteca/boletin- } \\
\text { de-novedades-educativas-n-60-entrevista-a-diana- } \\
\text { mazza-la-perspectiva-clinica-en-educacion }\end{array}$ \\
\hline $\begin{array}{l}\text { Algunas revisiones críticas en torno } \\
\text { a la noción del "triánoulo didáctico". }\end{array}$ & $\begin{array}{l}\text {-Alliaud, Andrea. (2017). } \\
\text {-Mazza Diana (2015) }\end{array}$ \\
\hline
\end{tabular}

